\title{
A Gaia oriented analysis of a large sample of quasars
}

\author{
A. H. Andrei ${ }^{1,2,3}$, M. Assafin ${ }^{2}$, C. Barache ${ }^{3}$, S. Bouquillon ${ }^{3}$, G. \\ Bourda $^{4}$, J. I. B. Camargo ${ }^{2}$, J.-F. le Campion ${ }^{4}$, P. Charlot ${ }^{4}$, A.-M. \\ Gontier $^{3}$, S. Lambert ${ }^{5}$, J.J. Pereira Osório ${ }^{6}$, D.N. da Silva Neto ${ }^{2,7}$, J. \\ Souchay $^{3}$ and R. Vieira Martins ${ }^{1}$ \\ ${ }^{1}$ Observatório Nacional/MCT \\ R. Gal. JosCristino 77, RJ, Br asil \\ email: oat1@on.br \\ ${ }^{2}$ Observatório do Valongo/UFRJ, Brasil \\ ${ }^{3}$ SYRTE/Observatoire de Paris, France \\ ${ }^{4}$ Observatoire de Bordeaux, France \\ ${ }^{5}$ Observatoire Royal de Belgique \\ ${ }^{6}$ Observatório Astronico da Universidade do Porto, Portugal \\ ${ }^{7}$ Universidade Estadual da Zona Oeste/RJ, Brasil
}

\begin{abstract}
Gaia photometric capabilities should distinguish quasars to a high degree of certainty. With this, they should also be able to deliver a clean sample of quasars with a negligible trace of stellar contaminants. However, a purely photometric sample could miss a non negligible percentage of ICRF sources counterparts - and this interface is required to align with the ICRS and de-rotate the GCRF (Gaia Celestial Reference Frame), on grounds of continuity. To prepare a minimum clean sample forming the initial quasar catalogue for the Gaia mission, an all sky ensemble was formed containing 128,257 candidates. Among them there is at least one redshift determination for $98.75 \%$, and at least one magnitude determination for $99.20 \%$ of the targets. The sources were collected from different optical and radio lists. We analyze the redshift, magnitude, and color distributions, their relationships, as well as their degree of completeness.

Complementary, the candidate sources enable to form an optical representation of the ICRS from first principles, namely, kinematically non-rotating with respect to the ensemble of distant extragalactic objects, aligned to the mean equator and dynamical equinox of J2000, and realized by a list of adopted coordinates of extragalactic sources.
\end{abstract}

Keywords. astronomical data bases: miscellaneous, astrometry, reference systems, quasars: general

\section{Gaia's Initial QSO catalogue and clean sample}

The establishment of an initial quasar catalogue for the Gaia mission must fulfill criteria of quantity and sky distribution. The expected number of quasars to be detected by Gaia is about 400,000 objects. That is about four times the number of QSOs presently known. A much smaller number, between 6,000 to 10,000, is required for definition of the fundamental frame. This sets the minimum requirements for the initial quasar catalogue clean sample, that is to zero level of contaminants. Since the all sky distribution (exempting the galactic disk) is also desirable, direct observations are out of question and the pre-existing quasar catalogues ought to be scrutinized. 
As the first step the common entries are sorted and a consolidated list is produced, including a reliability estimator and the radio astrometric accuracy. This consolidated list, plus information on redshift, multi-band magnitude and radio flux is the aim of the VLQAC (Souchay et al., 2007, and references therein)

Since the consolidated list in none of its constituents derives from apparent magnitude limits in the $\mathrm{G}$ band, a check must then be performed to test each source $\mathrm{G}$ magnitude. The B1.0 and GSC2.3 catalogs present the whole sky complete up to magnitude V=20 (though reaching beyond in some zones). For most of the sky they contain the $\mathrm{B}$, R, and I magnitudes, and so the G magnitude can be acceptably derived. With this the list is trimmed of its weaker sources (which will nevertheless be flagged as such and then kept), which would give rise to poor Gaia centroid determination.

Next, from available optical images, the objects PSF is compared to the stellar neighbors, in order to reject no pointlike objects. Finally, the objects observational history is followed, to the effect of retaining a core of (at least) double-checked quasars. The objects passing through all checks form the clean sample. They are further flagged according to their history of stable radio emission.

However, the clean sample does not meet the even sky distribution desirable. In order to densify the initial quasar list the sources failing to enter the clean sample are included, and accordingly flagged to, based on two additional tests. The assured quality of the single observation reported. The placement in the QSO color loci. These additional criteria involve a one-by-one examination of sources resulting to a slower pace of each decision. Currently about one thousand of objects are being examined.

\section{Optical celestial reference frame}

The construction of the OCRF (Optical Celestial Reference Frame, Andrei et al., 2007, and references therein) starts from the updated and presumably complete VLQAC (Very Large Quasar Catalog) list of QSOs, initial optical positions for those quasars are found in the USNO B1.0 and GSC 2.3 catalogues, and from the SDSS Data Release 5. The initial positions are next placed onto UCAC2 based reference frames, following by an aligment to the ICRF, as represented by the optical counterpart of ICRF sources as well as of the most precise sources from the VLBA calibrator list and from the VLA calibrator list. Finally the OCRF axis are surveyed through spherical harmonics, contemplating right ascension, declination and magnitude terms.

The OCRF contains J2000 refered equatorial coordinates of 105,000 quasars, well represented on all-sky basis, from -88.5 to +89.5 degree of declination, and with $0.5 \mathrm{deg}$ as the average distance between adjacent elements. The global aligment to the ICRF is of $1.5 \mathrm{mas}$, and the individual position accuracies are represented by $80 \mathrm{mas}+0.1 \mathrm{R}$ (where $\mathrm{R}$ is the red magnitude). As a by product, significant equatorial corrections appear for all the used catalogues (but the SDSS DR5); an empirical magnitude correcition can be considered for the GSC23 intermediate and faint magnitude range; both the 2MASS and the preliminary northernmost UCAC2 positions show consistent astrometric precision; and small harmonic terms.

\section{References}

Andrei, A. H., Souchay, J., Zacharias, N., Smart, R. L., de Cameargo, J. I. B., da Silva Neto, D. N., Vieira Martins, R., Assafin, M., Barache, C., Bouquillon, S., \& Penna, J. L. 2007, $A A$, in preparation.

Souchay, J., Andrei, A. H., Barache, C., Bouquillon, S., Suchet, D., Baudin, M., Gontier, A.-M., Lambert, S., Le Poncin-Lafitte, C., Taris, F., \& Arias, F. E. 2007, AA, in preparation. 